for its quality and value, with increase in its rating in the course of 10 years. Medical students of the Israeli Medical Corp. respond similarly to the programme and express a need for further training in communication with adolescents.

\section{P20 1 THE RELATIONSHIP BETWEEN A TRUSTED ADULT AND ADOLESCENT HEALTH AND EDUCATION OUTCOMES: A SYSTEMATIC REVIEW}

${ }^{1} \mathrm{R}$ Whitehead*, ${ }^{2} \mathrm{~J}$ Pringle, ${ }^{3} \mathrm{D}$ Milne, ${ }^{4} E$ Scott, ${ }^{2} \mathrm{~J}$ McAteer. ${ }^{1}$ Public Health Sciences, NHS Health Scotland, Glasgow, UK; ${ }^{2}$ Scottish Collaboration for Public Health Research and Policy, University of Edinburgh, Edinburgh, UK; ${ }^{3}$ Public Health, NHS Fife, Fife, UK; ${ }^{4}$ Children and Families Analysis, Scottish Government, Edinburgh, UK

\subsection{6/bmjpo-2019-RCPCH-SAHM.26}

Aims The presence of social networks is recognised to be a protective factor for adolescents' health and wellbeing, with the role of 'trusted adults' recently coming into sharper focus. There is, however, little review-level evidence concerning such relationships. Aims: 1) Identify what constitutes a trusted adult. 2) Evaluate the association between trusted adults and adolescent health/education outcomes. 3) Identify how to establish/maintain trusted adult relationships.

Methods Search terms (e.g., 'trusted adult', 'natural mentor', 'supportive adult') were used to query 13 bibliographic databases.Inclusion criteria: adolescents aged 10-19 years; role of trusted adult, defined as 'someone who children and young people may turn to for help, and will take them seriously'; reports health/educational outcomes; published between 01/01/ 07 and 31/12/17; English language. Exclusion criteria: parenting programmes; focus on populations with specific pre-existing health/learning conditions.

Results Of 2,908 retrieved articles, 192 met inclusion criteria. Most described primary quantitative studies (136 articles, including 14 randomised controlled trials) with 25 qualitative and 18 mixed-methods studies. Four meta-analyses, six systematic reviews, and three narrative reviews were also included. Whilst there exists no universal definition of the trusted adult role, commonly observed qualities include assistance with personal emotional problems, a close emotional bond and someone that 'makes an important positive difference'. Existing quantitative evidence provides an unclear picture of the association between trusted adult presence and adolescent outcomes, with reviews predominantly finding no overall effect or small effect sizes. A number of methodological issues were identified which may, in part, explain these modest and inconclusive findings. Chiefly, quantitative studies tend to use vague definitions of the trusted adult role. Qualitative studies are less ambiguous in this respect and tend to more frequently indicate a beneficial impact of the role. Barriers and facilitators to establishing a trusted adult relationship were identified. These suggest the youth work sector is particularly well placed as a setting to establish trusted adult relationships.

Conclusion Methodological limitations in existing literature mean it is difficult to make firm conclusions on the impact of the trusted adult role on adolescent outcomes. Recommendations are proposed for future research including the use of mixed-methods approaches.

\section{P21 SCHOOL-LEVEL PREDICTORS OF CONDUCT PROBLEMS TRAJECTORIES}

'LB Bevilacqua*, ${ }^{2}$ BDS De Stavola, ${ }^{2}$ RV Viner. ${ }^{1}$ UCL GOS Institute of Child Health, UCL Psychology and Language Sciences (Developmental Risk and Resilience Unit), London, UK; ${ }^{2}$ UCL GOS Institute of Child Health, University College London, London, UK

10.1136/bmjpo-2019-RCPCH-SAHM.27

Background and aims Conduct Problems (CP) can differ in terms of continuity/desistence across different stages of life such as childhood and adolescence. Although there have been a number of research efforts to investigate individual-level factors associated with continuity/desistence of CP in adolescence, less is known about school factors that may potentially underlie continuity/desistence of CP such as school-level factors. Our aim is to systematically investigate a number of schoollevel factors that may predict CP trajectories while controlling for individual-level factors.

Methods We run longitudinal latent class analysis (LLCA) to identify trajectories of CP across adolescence using the Learning Together study dataset. We then investigated the role of a number of school-level factors in predicting class membership using multinomial logistic regression. We identified two classes of CP: a stable low and a moderate-high class across males and females.

Results A number of school-level factors such as student-teachers' relationships, sense of belonging to the school and participation in school activities predicted persistent patterns of $\mathrm{CP}$ in males and females separately. Positive school atmosphere was found to be strongly associated with a lower risk of persistent CP across males and females.

Conclusions Student-teachers' relationships, sense of belonging to the school and participation in school activities are important aspects that researchers and schools should consider when implementing prevention and intervention programs for youth with $\mathrm{CP}$ and antisocial behaviour.

\section{P23 THE HEALTH OF MALAYSIA'S ADOLESCENTS: FINDINGS FROM THE GLOBAL BURDEN OF DISEASE 2017 STUDY}

${ }^{1} S$ Abdul-Razak* ${ }^{2,3,4} \mathrm{SM}$ Sawyer, ${ }^{2,3,4} \mathrm{GC}$ Patton, ${ }^{2} \mathrm{~K}$ Cini, ${ }^{5} \mathrm{NA}$ Ahmad, ${ }^{6} \mathrm{AH}$ Mokdad, 2,3,7PS Azzopardi. 'Department of Primary Care Medicine, Universiti Teknologi MARA, Selangor, Malaysia; ${ }^{2}$ Centre for Adolescent Health, Royal Children's Hospital and Murdoch Children's Research Institute, Parkville, Victoria, Australia; ${ }^{3}$ Department of Paediatrics, The University of Melbourne, Parkville, Victoria, Australia; ${ }^{4}$ Nossal Institute, School of Population and Global Health, The University of Melbourne, Parkville, Victoria, Australia; ${ }^{5}$ Institute for Public Health, Ministry of Health Malaysia, Kuala Lumpur, Malaysia; ${ }^{6}$ Institute for Health Metrics and Evaluation, University of Washington, Seattle, Washington, USA; ${ }^{7}$ Burnet Institute, Melbourne, Australia

\subsection{6/bmjpo-2019-RCPCH-SAHM.28}

Aim The epidemiological transition has resulted in a large population of adolescents in Malaysia, whose health burden is poorly described. We set out to provide a comprehensive profile of health in Malaysian adolescents to illustrate important targets for health actions.

Methods A conceptual framework for reporting health and wellbeing of Malaysian adolescents was defined to measure health outcomes, health risks and sociocultural determinants. Data from the Global Burden of Disease 2017 study were used to analyse mortality (all-cause and cause-specific 